Article

\title{
Strong Solutions of the Incompressible Navier-Stokes-Voigt Model
}

\author{
Evgenii S. Baranovskii
}

Department of Applied Mathematics, Informatics and Mechanics, Voronezh State University, 394018 Voronezh, Russia; esbaranovskii@gmail.com

Received: 30 December 2019; Accepted: 28 January 2020; Published: 3 February 2020

\begin{abstract}
This paper deals with an initial-boundary value problem for the Navier-Stokes-Voigt equations describing unsteady flows of an incompressible non-Newtonian fluid. We give the strong formulation of this problem as a nonlinear evolutionary equation in Sobolev spaces. Using the Faedo-Galerkin method with a special basis of eigenfunctions of the Stokes operator, we construct a global-in-time strong solution, which is unique in both two-dimensional and three-dimensional domains. We also study the long-time asymptotic behavior of the velocity field under the assumption that the external forces field is conservative.
\end{abstract}

Keywords: Navier-Stokes-Voigt equations; viscoelastic models; non-Newtonian fluid; strong solutions; existence and uniqueness theorem; Faedo-Galerkin approximations; Stokes operator; long-time behavior

\section{Introduction}

In this work, we study an initial-boundary value problem for the Navier-Stokes-Voigt (NSV) equations that model the unsteady flow of an incompressible viscoelastic fluid:

$$
\left\{\begin{array}{l}
\frac{\partial \boldsymbol{u}}{\partial t}+(\boldsymbol{u} \cdot \nabla) \boldsymbol{u}-v \Delta \boldsymbol{u}-\alpha^{2} \frac{\partial \Delta \boldsymbol{u}}{\partial t}+\nabla p=f \text { in } \Omega \times(0,+\infty), \\
\nabla \cdot \boldsymbol{u}=0 \text { in } \Omega \times(0,+\infty), \\
\boldsymbol{u}=\mathbf{0} \text { on } \partial \Omega \times(0,+\infty), \\
\boldsymbol{u}(\cdot, 0)=\boldsymbol{u}_{0} \text { in } \Omega,
\end{array}\right.
$$

where $\Omega$ denotes the bounded domain of flow in $\mathbb{R}^{n}, n=2$, 3, with boundary $\partial \Omega$; the vector function $u$ represents the velocity field; $p$ denotes the pressure; $v>0$ is the viscosity coefficient; $\alpha$ is a length scale parameter such that $\alpha^{2} / v$ is the relaxation time of the viscoelastic fluid; $f$ is the external forces field; and $\boldsymbol{u}_{0}$ is the initial velocity.

Note that when $\alpha=0$ the NSV system becomes the incompressible Navier-Stokes equations that describe Newtonian fluid flows. If $\alpha=0$ and $v=0$, then we arrive at the incompressible Euler equations governing inviscid flows.

In the literature, the NSV equations are often called the Kelvin-Voigt equations or Oskolkov's equations. The NSV model and related models of viscoelastic fluid flows have been studied extensively by different mathematicians over the past several decades starting from the pioneering papers by Oskolkov [1,2]. It should be mentioned at this point that Oskolkov later admitted [3] that these works contain some errors and not all obtained results hold. In this regard, Ladyzhenskaya remarked in her note [4] that the method of introduction of auxiliary viscosity used in [1,2] is incorrect under the 
no-slip boundary condition and explained the reasons for this. However, it is certain that the series of Oskolkov's works played a major role in the study of the NSV equations and stimulated further research in this direction.

Let us shortly review available literature on mathematical analysis of NSV-type models. Sviridyuk [5] established the solvability of the weakly compressible NSV equations. In [6], the local-in-time unique solvability of problem (1) is proved. Korpusov and Sveshnikov [7] investigated the blowup of solutions to the NSV equations with a cubic source. Various slip problems are studied in the papers [8-10]. Kaya and Celebi [11] proved the existence and uniqueness of weak solutions of the so-called g-Kelvin-Voigt equations that describe viscoelastic fluid flows in thin domains. The solvability of the inhomogeneous Dirichlet problem for the equations governing a polymer fluid flow is proved in [12]. Berselli and Spirito [13] showed that weak solutions to the Navier-Stokes equations obtained as limits $\alpha \rightarrow 0^{+}$of solutions to the NSV model are "suitable weak solutions" [14] and satisfy the local energy inequality. Fedorov and Ivanova [15] dealt with an inverse problem for the NSV equations. An algorithm for finding of numerical solution of an optimal control problem for the two-dimensional Kelvin-Voigt fluid flow was proposed by Plekhanova et al. [16]. Antontsev and Khompysh [17] established the existence and uniqueness of the global and local weak solutions to the NSV equations with p-Laplacian and a damping term. Artemov and Baranovskii [18] proved the existence of weak solutions to the coupled system of nonlinear equations describing the heat transfer in steady-state flows of a polymeric fluid. Mohan [19] investigated the global solvability, the asymptotic behavior, and some control problems for the NSV model with "fading memory" and "memory of length $\tau^{\prime \prime}$.

Most of the papers mentioned above deal with the study of weak (generalized) solutions to the NSV equations in the framework of the Hilbert space techniques. Therefore, it is a relevant question to prove the existence and uniqueness of strong solutions of system (1) in a Banach space under natural conditions on the data. Another important objective is to develop convenient algorithms for finding strong solutions or their approximations. Motivated by this, in the present work, we propose the strong formulation of problem (1) as a nonlinear evolutionary equation in suitable Banach spaces with the initial condition $\boldsymbol{u}(0)=\boldsymbol{u}_{0}$. Using the Faedo-Galerkin procedure with a special basis of eigenfunctions of the Stokes operator and deriving various a priori estimates of approximate solutions in Sobolev's spaces $\boldsymbol{H}^{1}(\Omega)$ and $\boldsymbol{H}^{2}(\Omega)$, we construct a global-in-time strong solution of (1), which is unique in both two-dimensional and three-dimensional domains. We also derive the energy equality that holds for strong solutions. Moreover, it is shown that, if the external forces field $f$ is conservative, then the $H^{1}$-norm of the velocity field $u$ decays exponentially as $t \rightarrow+\infty$.

\section{Preliminaries}

To suggest the concept of a strong solution to problem (1), we introduce some notations, function spaces, and auxiliary results.

For vectors $x, y \in \mathbb{R}^{n}$ and matrices $\mathrm{X}, \mathrm{Y} \in \mathbb{R}^{n \times n}$ by $\boldsymbol{x} \cdot \boldsymbol{y}$ and $\mathrm{X}: \mathrm{Y}$, we denote the scalar products, respectively:

$$
\boldsymbol{x} \cdot \boldsymbol{y} \stackrel{\text { def }}{=} \sum_{i=1}^{n} x_{i} y_{i}, \quad \mathbf{X}: \mathbf{Y} \stackrel{\text { def }}{=} \sum_{i, j=1}^{n} X_{i j} Y_{i j}
$$

Let $\Omega \subset \mathbb{R}^{n}$ be a bounded domain with sufficiently smooth boundary $\partial \Omega$. By $\mathcal{D}(\Omega)$ denote the set of $\mathcal{C}^{\infty}$ functions with support contained in $\Omega$. We use the standard notation for the Lebesgue spaces $L^{S}(\Omega), s \geq 1$, as well as the Sobolev spaces $H^{k}(\Omega) \stackrel{\text { def }}{=} W^{k, 2}(\Omega), k \in \mathbb{N}$. When it comes to classes of $\mathbb{R}^{n}$-valued functions, we employ boldface letters, for instance,

$$
\boldsymbol{D}(\Omega) \stackrel{\text { def }}{=} \mathcal{D}(\Omega)^{n}, \quad \boldsymbol{L}^{s}(\Omega) \stackrel{\text { def }}{=} L^{s}(\Omega)^{n}, \quad \boldsymbol{H}^{k}(\Omega) \stackrel{\text { def }}{=} H^{k}(\Omega)^{n} .
$$

It is well known that the space Sobolev $H^{1}(\Omega)$ is compactly embedded in $L^{4}(\Omega)$. 
Let us introduce the following spaces:

$$
\begin{aligned}
& \mathcal{V}(\Omega) \stackrel{\text { def }}{=}\{v \in D(\Omega): \nabla \cdot v=0\}, \\
& V^{0}(\Omega) \stackrel{\text { def }}{=} \text { the closure of the set } \mathcal{V}(\Omega) \text { in the space } L^{2}(\Omega), \\
& V^{1}(\Omega) \stackrel{\text { def }}{=} \text { the closure of the set } \mathcal{V}(\Omega) \text { in the space } H^{1}(\Omega), \\
& V^{2}(\Omega) \stackrel{\text { def }}{=} H^{2}(\Omega) \cap V^{1}(\Omega) .
\end{aligned}
$$

It is obvious that $V^{0}(\Omega), V^{1}(\Omega)$, and $V^{2}(\Omega)$ are Hilbert spaces with the scalar products induced by $\boldsymbol{L}^{2}(\Omega), \boldsymbol{H}^{1}(\Omega)$, and $\boldsymbol{H}^{2}(\Omega)$, respectively. However, when studying problem (1), in the spaces $\boldsymbol{V}^{1}(\Omega)$ and $V^{2}(\Omega)$, it is more convenient to use the scalar products and the norms defined as follows:

$$
\begin{aligned}
& (\boldsymbol{v}, \boldsymbol{w})_{V^{1}(\Omega)} \stackrel{\text { def }}{=}(\boldsymbol{v}, \boldsymbol{w})_{L^{2}(\Omega)}+\alpha^{2}(\nabla \boldsymbol{v}, \nabla \boldsymbol{w})_{L^{2}(\Omega)^{\prime}} \quad\|\boldsymbol{v}\|_{V^{1}(\Omega)} \stackrel{\text { def }}{=}(\boldsymbol{v}, \boldsymbol{v})_{V^{1}(\Omega)^{\prime}}^{1 / 2} \\
& (\boldsymbol{v}, \boldsymbol{w})_{V^{2}(\Omega)} \stackrel{\text { def }}{=}(\mathbb{P} \Delta \boldsymbol{v}, \mathbb{P} \Delta \boldsymbol{w})_{L^{2}(\Omega)}, \quad\|\boldsymbol{v}\|_{V^{2}(\Omega)} \stackrel{\text { def }}{=}(\boldsymbol{v}, \boldsymbol{v})_{V^{2}(\Omega)}^{1 / 2} \text {. }
\end{aligned}
$$

Here, $\mathbb{P}: L^{2}(\Omega) \rightarrow V^{0}(\Omega)$ is the Leray projection, which corresponds the well-known Leray (or Hodge-Helmholtz) decomposition for the vector fields in $L^{2}(\Omega)$ into a divergence-free part and a gradient part (see, e.g., [20], Chapter IV):

$$
L^{2}(\Omega)=V^{0}(\Omega) \oplus G(\Omega),
$$

where the symbol $\oplus$ denotes the orthogonal sum and the subspace $G(\Omega)$ is defined as follows

$$
G(\Omega) \stackrel{\text { def }}{=}\left\{\nabla h: h \in H^{1}(\Omega)\right\} .
$$

Note that the norm $\|\cdot\|_{V^{i}(\Omega)}$ is equivalent to the norm $\|\cdot\|_{H^{i}(\Omega)}, i=1,2$.

We introduce the equivalence relation on the space $H^{1}(\Omega)$ by stating that $\varphi \sim \psi$ if $\varphi-\psi=$ const. As usual, $H^{1}(\Omega) / \mathbb{R}$ denotes the quotient of $H^{1}(\Omega)$ by $\mathbb{R}$.

For a function $\xi \in H^{1}(\Omega)$, we set

$$
\bar{\xi} \stackrel{\text { def }}{=}\left\{\omega \in H^{1}(\Omega): \omega \sim \xi\right\} \in H^{1}(\Omega) / \mathbb{R} .
$$

Let us define the gradient and the norm of $\bar{\xi}$ as follows

$$
\nabla \bar{\xi} \stackrel{\text { def }}{=} \nabla \xi, \quad\|\bar{\xi}\|_{H^{1}(\Omega) / \mathbb{R}} \stackrel{\text { def }}{=}\|\nabla \bar{\xi}\|_{L^{2}(\Omega)} .
$$

Using Proposition 1.2 from ([21], Chapter I, §1), it is easy to verify that the norm $\|\cdot\|_{H^{1}(\Omega) / \mathbb{R}}$ is well defined.

The following lemmas are needed for the sequel.

Lemma 1. Suppose $\boldsymbol{E}$ is a Banach space and $T$ is a positive number. A set $\boldsymbol{K}$ of the space $\boldsymbol{C}([0, T] ; \boldsymbol{E})$ is relatively compact if and only if:

- for any number $t \in(0, T)$, the set $\boldsymbol{K}(t) \stackrel{\text { def }}{=}\{\boldsymbol{w}(t): \boldsymbol{w} \in \boldsymbol{K}\}$ is relatively compact in $\boldsymbol{E}$;

- for any number $\varepsilon>0$, there exists a number $\eta>0$ such that the inequality

$$
\left\|\boldsymbol{w}\left(t_{1}\right)-\boldsymbol{w}\left(t_{2}\right)\right\|_{E} \leq \varepsilon
$$

holds for any function $\boldsymbol{w} \in \mathbf{K}$ and any numbers $t_{1}, t_{2} \in[0, T]$ such that $\left|t_{1}-t_{2}\right| \leq \eta$.

The proof of this lemma is given in [22]. 
Lemma 2. The embedding $C^{1}\left([0, T] ; V^{2}(\Omega)\right) \hookrightarrow C\left([0, T] ; V^{1}(\Omega)\right)$ is completely continuous.

Proof. Let $S$ be a bounded set of $C^{1}\left([0, T] ; V^{2}(\Omega)\right)$. Then

$$
\max _{(\boldsymbol{w}, t) \in S \times[0, T]}\|\boldsymbol{w}(t)\|_{V^{2}(\Omega)}+\max _{(\boldsymbol{w}, t) \in S \times[0, T]}\left\|\boldsymbol{w}^{\prime}(t)\right\|_{V^{2}(\Omega)} \leq r
$$

with some constant $r$. Clearly, this implies that the set

$$
S(t) \stackrel{\text { def }}{=}\{\boldsymbol{w}(t): \boldsymbol{w} \in S\}
$$

is bounded in $V^{2}(\Omega)$ for any $t \in[0, T]$.

From the Rellich-Kondrachov theorem (see, e.g., [23], Chapter 1, Theorem 1.12.1), it follows that the space $V^{2}(\Omega)$ is compactly embedded into $V^{1}(\Omega)$. Therefore, the set $S(t)$ is relatively compact in the space $V^{1}(\Omega)$.

By $I$ denote the embedding operator from $V^{2}(\Omega)$ into $V^{1}(\Omega)$. Taking into account inequality (5), we get the estimate

$$
\begin{aligned}
\left\|\boldsymbol{w}\left(t_{1}\right)-\boldsymbol{w}\left(t_{2}\right)\right\|_{V^{1}(\Omega)} & \leq\|\mathbf{I}\|_{\mathcal{L}\left(V^{2}(\Omega), V^{1}(\Omega)\right)}\left\|\boldsymbol{w}\left(t_{1}\right)-\boldsymbol{w}\left(t_{2}\right)\right\|_{V^{2}(\Omega)} \\
& \leq\|\mathbf{I}\|_{\mathcal{L}\left(V^{2}(\Omega), V^{1}(\Omega)\right)} \max _{\tau \in\left[t_{1}, t_{2}\right]}\left\|\boldsymbol{w}^{\prime}(\tau)\right\|_{V^{2}(\Omega)}\left|t_{1}-t_{2}\right| \\
& \leq r\|\mathbf{I}\|_{\mathcal{L}\left(V^{2}(\Omega), V^{1}(\Omega)\right)}\left|t_{1}-t_{2}\right| \leq \varepsilon
\end{aligned}
$$

for any function $w \in S$ and for any numbers $t_{1}, t_{2} \in[0, T]$ such that

$$
\left|t_{1}-t_{2}\right| \leq \frac{\varepsilon}{r\|\mathbf{I}\|_{\mathcal{L}\left(V^{2}(\Omega), V^{1}(\Omega)\right)}}
$$

where $\|\mathbf{I}\|_{\mathcal{L}\left(V^{2}(\Omega), V^{1}(\Omega)\right)}$ is the operator norm of $\mathbf{I}$.

Applying Lemma 1 with $E=V^{1}(\Omega)$, we conclude that the set $S$ is relatively compact in the space $C\left([0, T] ; V^{1}(\Omega)\right)$. Lemma 2 is proved.

Lemma 3. Let

$$
\mathfrak{L}_{d} \stackrel{\text { def }}{=}\left\{x \in \mathbb{R}^{n}:\left|x_{n}\right|<d / 2\right\}, \quad d_{\Omega} \stackrel{\text { def }}{=} \inf \left\{d>0: \Omega \subset \mathfrak{L}_{d}\right\} .
$$

Then, we have

$$
\frac{4}{d_{\Omega}^{2}+4 \alpha^{2}}\left(\|v\|_{L^{2}(\Omega)}^{2}+\alpha^{2}\|\nabla v\|_{L^{2}(\Omega)}^{2}\right) \leq\|\nabla v\|_{L^{2}(\Omega)^{\prime}}^{2}
$$

for any $v \in V^{1}(\Omega)$.

Proof. The estimate (6) is a direct consequence of the Poincaré inequality (see, e.g., [24], Chapter II, Theorem II.5.1).

\section{Strong Formulation of Problem (1) and Main Results}

Let us suppose that

$$
f \in C\left([0,+\infty) ; L^{2}(\Omega)\right), \quad u_{0} \in V^{2}(\Omega) .
$$

Definition 1. We say that a pair $(\boldsymbol{u}, \bar{p})$ is a strong solution to problem (1) if

$$
\boldsymbol{u} \in C^{1}\left([0,+\infty) ; \boldsymbol{V}^{2}(\Omega)\right), \quad \bar{p} \in C\left([0,+\infty) ; H^{1}(\Omega) / \mathbb{R}\right)
$$


and the following equalities are valid:

$$
\begin{gathered}
\boldsymbol{u}^{\prime}(t)+(\boldsymbol{u}(t) \cdot \nabla) \boldsymbol{u}(t)-v \Delta \boldsymbol{u}(t)-\alpha^{2} \Delta \boldsymbol{u}^{\prime}(t)+\nabla \bar{p}(t)=f(t), \quad t>0 \\
\boldsymbol{u}(0)=\boldsymbol{u}_{0} .
\end{gathered}
$$

Remark 1. Equation (8) with the initial condition $\boldsymbol{u}(0)=\boldsymbol{u}_{0}$ is a natural interpretation of the initial-boundary value problem (1) as an evolutionary equation in suitable function spaces. Note that, if a pair $\left(\boldsymbol{u}_{*}, p_{*}\right)$ is a classical solution to problem (1), then $\left(\boldsymbol{u}_{*}, \bar{p}_{*}\right)$ satisfies Equation (8), i.e., this pair is a strong solution. On the other hand, if $(\boldsymbol{u}, \bar{p})$ is a strong solution and the functions $\boldsymbol{u}$ and $p$ are sufficiently smooth in the usual sense, then $(\boldsymbol{u}, p)$ is a classical solution to (1).

We are now in a position to state our main results.

Theorem 1. Assume that the boundary of the domain $\Omega$ belongs to the class $\mathcal{C}^{2}$ and condition (7) holds. Then problem (1) has a unique strong solution $(\boldsymbol{u}, \bar{p})$. This strong solution satisfies the energy equality

$$
\begin{aligned}
\|\boldsymbol{u}(t)\|_{L^{2}(\Omega)}^{2}+2 v \int_{0}^{t}\|\nabla \boldsymbol{u}(\tau)\|_{\mathbf{L}^{2}(\Omega)}^{2} d \tau+\alpha^{2}\|\nabla \boldsymbol{u}(t)\|_{\mathbf{L}^{2}(\Omega)}^{2} & \\
& =\left\|\boldsymbol{u}_{0}\right\|_{\mathbf{L}^{2}(\Omega)}^{2}+\alpha^{2}\left\|\nabla \boldsymbol{u}_{0}\right\|_{\mathbf{L}^{2}(\Omega)}^{2}+2 \int_{0}^{t} \int_{\Omega} \boldsymbol{f}(\tau) \cdot \boldsymbol{u}(\tau) d x d \tau, \quad t \geq 0 .
\end{aligned}
$$

If there exists a function $q \in C\left([0, T] ; H^{1}(\Omega)\right)$ such that $\nabla q=f$, then

$$
\|\boldsymbol{u}(t)\|_{L^{2}(\Omega)}^{2}+\alpha^{2}\|\nabla \boldsymbol{u}(t)\|_{L^{2}(\Omega)}^{2} \leq \exp \left(-\frac{8 v t}{d_{\Omega}^{2}+4 \alpha^{2}}\right)\left(\left\|\boldsymbol{u}_{0}\right\|_{L^{2}(\Omega)}^{2}+\alpha^{2}\left\|\nabla \boldsymbol{u}_{0}\right\|_{L^{2}(\Omega)}^{2}\right), \quad t \geq 0,
$$

where the positive constant $d_{\Omega}$ is defined in Lemma 3.

\section{Proof of Theorem 1}

To prove the existence of a strong solution to problem (1), we use the Faedo-Galerkin method with a special basis of eigenfunctions of the Stokes operator

$$
\mathbb{A}: V^{2}(\Omega) \rightarrow V^{0}(\Omega), \quad \mathbb{A} w \stackrel{\text { def }}{=}-\mathbb{P} \Delta w .
$$

This linear operator is invertible and $\mathbb{A}^{-1}$ is self-adjoint and compact as a map from $V^{0}(\Omega)$ into $V^{0}(\Omega)$. From the spectral theorem for self-adjoint compact operators (see, e.g., [25], Chapter 10, Theorem 10.12), it follows that there exist sequences $\left\{\boldsymbol{w}_{j}\right\}_{j=1}^{\infty} \subset \boldsymbol{V}^{2}(\Omega)$ and $\left\{\lambda_{j}\right\}_{j=1}^{\infty} \subset(0,+\infty)$ such that

$$
\mathbb{A} \boldsymbol{w}_{j}=\lambda_{j} \boldsymbol{w}_{j}, \quad j \in\{1,2, \ldots\},
$$

and $\left\{\boldsymbol{w}_{j}\right\}_{j=1}^{\infty}$ is an orthonormal basis of the space $\boldsymbol{V}^{0}(\Omega)$.

Let

$$
\widetilde{\boldsymbol{w}}_{j} \stackrel{\text { def }}{=} \lambda_{j}^{-1} \boldsymbol{w}_{j}, \quad j \in\{1,2, \ldots\} .
$$

It is easily shown that $\left\{\widetilde{\boldsymbol{w}}_{j}\right\}_{j=1}^{\infty}$ is an orthonormal basis in the space $V^{2}(\Omega)$.

Let us fix an arbitrary number $T>0$. For each fixed integer $m \geq 1$, we would like to define the approximate solution as follows:

$$
\boldsymbol{v}_{m}(t) \stackrel{\text { def }}{=} \sum_{i=1}^{m} g_{m i}(t) \boldsymbol{w}_{i}, \quad t \in[0, T]
$$


where $g_{m 1}, \ldots, g_{m m}$ are unknown functions such that

$$
\left\{\begin{array}{l}
\int_{\Omega} \boldsymbol{v}_{m}^{\prime}(t) \cdot \boldsymbol{w}_{j} d x+\sum_{i=1}^{n} \int_{\Omega} v_{m i}(t) \frac{\partial \boldsymbol{v}_{m}(t)}{\partial x_{i}} \cdot \boldsymbol{w}_{j} d x-v \int_{\Omega} \Delta \boldsymbol{v}_{m}(t) \cdot \boldsymbol{w}_{j} d x \\
-\alpha^{2} \int_{\Omega} \Delta \boldsymbol{v}_{m}^{\prime}(t) \cdot \boldsymbol{w}_{j} d x=\int_{\Omega} f(t) \cdot \boldsymbol{w}_{j} d x, \quad t \in(0, T), j=1, \ldots, m, \\
\boldsymbol{v}_{m}(0)=\sum_{i=1}^{m}\left(\boldsymbol{u}_{0}, \widetilde{\boldsymbol{w}}_{i}\right)_{V^{2}(\Omega)} \widetilde{\boldsymbol{w}}_{i} .
\end{array}\right.
$$

Let us define the matrix $Q_{m} \in \mathbb{R}^{m \times m}$ and the vector $\boldsymbol{a}_{m} \in \mathbb{R}^{m}$ by the rules:

$$
\begin{aligned}
Q_{m i j} & \stackrel{\text { def }}{=} \int_{\Omega} w_{i} \cdot w_{j} d x-\alpha^{2} \int_{\Omega} \Delta w_{i} \cdot w_{j} d x, \quad i, j=1, \ldots, m, \\
a_{m i} & \stackrel{\text { def }}{=} \lambda_{i}^{-2}\left(\boldsymbol{u}_{0}, \boldsymbol{w}_{i}\right)_{V^{2}(\Omega)}, \quad i=1, \ldots, m .
\end{aligned}
$$

Then, system (12) can be rewritten in the form

$$
\left\{\begin{array}{l}
\boldsymbol{Q}_{m}^{\top} \boldsymbol{g}_{m}^{\prime}(t)=\mathbf{F}_{m}\left(t, \boldsymbol{g}_{m}(t)\right), \quad t \in(0, T), \\
\boldsymbol{g}_{m}(0)=\boldsymbol{a}_{m},
\end{array}\right.
$$

where $\mathbf{F}_{m}:[0, T] \times \mathbb{R}^{m} \rightarrow \mathbb{R}^{m}$ is a known nonlinear vector function and $\boldsymbol{g}_{m} \stackrel{\text { def }}{=}\left(g_{m 1}, \ldots, g_{m m}\right)$.

Using integration by parts, we obtain

$$
Q_{m i j}=\left(\boldsymbol{w}_{i}, \boldsymbol{w}_{j}\right)_{V^{1}(\Omega)}, \quad i, j=1, \ldots, m .
$$

Therefore, the matrix $Q_{m}$ is symmetric and invertible.

Applying $Q_{m}^{-1}$ to the first equation of problem (13), we obviously get

$$
\left\{\begin{array}{l}
\boldsymbol{g}_{m}^{\prime}(t)=Q_{m}^{-1} \mathbf{F}_{m}\left(t, \boldsymbol{g}_{m}(t)\right), \quad t \in(0, T), \\
\boldsymbol{g}_{m}(0)=\boldsymbol{a}_{m} .
\end{array}\right.
$$

The local existence of $\boldsymbol{g}_{m}$ on an interval $\left[0, T_{m}\right]$ is insured by the Cauchy-Peano theorem. Thus, we have a local solution $\boldsymbol{v}_{m}$ of problem (12) on $\left[0, T_{m}\right]$. Below, we obtain a priori estimates (independent of $m$ ) for vector function $v_{m}$, which entail that $T_{m}=T$.

Let us assume that $\boldsymbol{v}_{m}$ satisfies system (12). We multiply the $j$ th equation of (12) by $g_{m j}(t)$ and sum with respect to $j$ from 1 to $m$. Since

$$
\begin{aligned}
\sum_{i=1}^{n} \int_{\Omega} v_{m i}(t) \frac{\partial v_{m}(t)}{\partial x_{i}} \cdot v_{m}(t) d x & =\frac{1}{2} \sum_{i=1}^{n} \int_{\Omega} v_{m i}(t) \frac{\partial}{\partial x_{i}}\left|\boldsymbol{v}_{m}(t)\right|^{2} d x \\
& =-\frac{1}{2} \sum_{i=1}^{n} \int_{\Omega} \frac{\partial v_{m i}(t)}{\partial x_{i}}\left|\boldsymbol{v}_{m}(t)\right|^{2} d x \\
& =-\frac{1}{2} \int \underbrace{\nabla \cdot \boldsymbol{v}_{m}(t)}_{=0}\left|\boldsymbol{v}_{m}(t)\right|^{2} d x \\
& =0, \quad t \in(0, T),
\end{aligned}
$$


we get

$$
\int_{\Omega} \boldsymbol{v}_{m}^{\prime}(t) \cdot \boldsymbol{v}_{m}(t) d x-v \int_{\Omega} \Delta \boldsymbol{v}_{m}(t) \cdot \boldsymbol{v}_{m}(t) d x-\alpha^{2} \int_{\Omega} \Delta \boldsymbol{v}_{m}^{\prime}(t) \cdot \boldsymbol{v}_{m}(t) d x=\int_{\Omega} f(t) \cdot \boldsymbol{v}_{m}(t) d x
$$

Integrating by parts the second and third terms on the left-hand side of equality (14), we arrive at the following relation

$$
\int_{\Omega} \boldsymbol{v}_{m}^{\prime}(t) \cdot \boldsymbol{v}_{m}(t) d x+v \int_{\Omega}\left|\nabla \boldsymbol{v}_{m}(t)\right|^{2} d x+\alpha^{2} \int_{\Omega} \nabla \boldsymbol{v}_{m}^{\prime}(t): \nabla \boldsymbol{v}_{m}(t) d x=\int_{\Omega} f(t) \cdot \boldsymbol{v}_{m}(t) d x,
$$

which, in turn, gives

$$
\frac{1}{2} \frac{d}{d \tau}\left\|\boldsymbol{v}_{m}(\tau)\right\|_{L^{2}(\Omega)}^{2}+v\left\|\nabla \boldsymbol{v}_{m}(\tau)\right\|_{L^{2}(\Omega)}^{2}+\frac{\alpha^{2}}{2} \frac{d}{d \tau}\left\|\nabla \boldsymbol{v}_{m}(\tau)\right\|_{L^{2}(\Omega)}^{2}=\int_{\Omega} f(\tau) \cdot \boldsymbol{v}_{m}(\tau) d x,
$$

for any $\tau \in[0, T]$. Further, we multiply the last equality by 2 and integrate from 0 to $t$ with respect to $\tau$; this yields

$$
\begin{aligned}
\left\|\boldsymbol{v}_{m}(t)\right\|_{L^{2}(\Omega)}^{2}+2 v \int_{0}^{t}\left\|\nabla \boldsymbol{v}_{m}(\tau)\right\|_{L^{2}(\Omega)}^{2} d \tau+\alpha^{2}\left\|\nabla \boldsymbol{v}_{m}(t)\right\|_{L^{2}(\Omega)}^{2} & \\
& =\left\|\boldsymbol{v}_{m}(0)\right\|_{L^{2}(\Omega)}^{2}+\alpha^{2}\left\|\nabla \boldsymbol{v}_{m}(0)\right\|_{L^{2}(\Omega)}^{2}+2 \int_{0}^{t} \int_{\Omega} f(\tau) \cdot \boldsymbol{v}_{m}(\tau) d x d \tau .
\end{aligned}
$$

Taking into account (3) and (4), we easily derive from equality (15) that

$$
\begin{aligned}
\left\|\boldsymbol{v}_{m}(t)\right\|_{\boldsymbol{V}^{1}(\Omega)}^{2} & \leq\left\|\boldsymbol{v}_{m}(0)\right\|_{V^{1}(\Omega)}^{2}+2 \int_{0}^{t} \int_{\Omega} f(\tau) \cdot \boldsymbol{v}_{m}(\tau) d x d \tau \\
& \leq\left\|\boldsymbol{v}_{m}(0)\right\|_{V^{1}(\Omega)}^{2}+\int_{0}^{t} \int_{\Omega}|f(\tau)|^{2} d x d \tau+\int_{0}^{t} \int_{\Omega}\left|\boldsymbol{v}_{m}(\tau)\right|^{2} d x d \tau \\
& \leq C_{1}\left\|\boldsymbol{u}_{0}\right\|_{V^{2}(\Omega)}^{2}+\int_{0}^{T}\|f(\tau)\|_{L^{2}(\Omega)}^{2} d \tau+C_{2} \int_{0}^{t}\left\|\boldsymbol{v}_{m}(\tau)\right\|_{V^{1}(\Omega)}^{2} d \tau .
\end{aligned}
$$

Here and in the succeeding discussion, the symbols $C_{i}, i=1,2, \ldots$, designate positive constants that are independent of $m$. Using Grönwall's inequality, we get

$$
\left\|\boldsymbol{v}_{m}(t)\right\|_{\boldsymbol{V}^{1}(\Omega)}^{2} \leq\left(C_{1}\left\|\boldsymbol{u}_{0}\right\|_{\boldsymbol{V}^{2}(\Omega)}^{2}+\int_{0}^{T}\|f(\tau)\|_{L^{2}(\Omega)}^{2} d \tau\right) \exp \left(C_{2} t\right), \quad t \in(0, T)
$$

Hence,

$$
\begin{aligned}
\left\|\boldsymbol{v}_{m}\right\|_{\boldsymbol{C}\left([0, T] ; \boldsymbol{V}^{1}(\Omega)\right)} & =\max _{t \in[0, T]}\left\|\boldsymbol{v}_{m}(t)\right\|_{V^{1}(\Omega)} \\
& \leq\left(C_{1}\left\|\boldsymbol{u}_{0}\right\|_{V^{2}(\Omega)}^{2}+\int_{0}^{T}\|f(\tau)\|_{L^{2}(\Omega)}^{2} d \tau\right)^{1 / 2}\left[\exp \left(C_{2} T\right)\right]^{1 / 2}
\end{aligned}
$$


Next, by multiplying the $j$ th equation of (12) with $g_{m j}^{\prime}$ and summing over $j=1, \ldots, m$, we obtain

$$
\begin{aligned}
\int_{\Omega}\left|\boldsymbol{v}_{m}^{\prime}(t)\right|^{2} d x+\sum_{i=1}^{n} \int_{\Omega} v_{m i}(t) \frac{\partial v_{m}(t)}{\partial x_{i}} \cdot v_{m}^{\prime}(t) d x-v \int_{\Omega} \Delta \boldsymbol{v}_{m}(t) \cdot \boldsymbol{v}_{m}^{\prime}(t) d x & \\
& -\alpha^{2} \int_{\Omega} \Delta v_{m}^{\prime}(t) \cdot \boldsymbol{v}_{m}^{\prime}(t) d x=\int_{\Omega} f(t) \cdot \boldsymbol{v}_{m}^{\prime}(t) d x, \quad t \in(0, T) .
\end{aligned}
$$

Integrating by parts the third and fourth terms on the left-hand side of the last equality, we arrive at

$$
\begin{aligned}
\int_{\Omega}\left|\boldsymbol{v}_{m}^{\prime}(t)\right|^{2} d x+\sum_{i=1}^{n} \int_{\Omega} v_{m i}(t) \frac{\partial v_{m}(t)}{\partial x_{i}} \cdot \boldsymbol{v}_{m}^{\prime}(t) & d x+v \int_{\Omega} \nabla \boldsymbol{v}_{m}(t): \nabla \boldsymbol{v}_{m}^{\prime}(t) d x \\
& +\alpha^{2} \int_{\Omega}\left|\nabla \boldsymbol{v}_{m}^{\prime}(t)\right|^{2} \boldsymbol{d} x=\int_{\Omega} f(t) \cdot \boldsymbol{v}_{m}^{\prime}(t) d x, \quad t \in(0, T) .
\end{aligned}
$$

From here, using (3) and Hölder's inequality, one can obtain

$$
\begin{aligned}
\left\|\boldsymbol{v}_{m}^{\prime}(t)\right\|_{\boldsymbol{V}^{1}(\Omega)}^{2}= & -\sum_{i=1}^{n} \int_{\Omega} v_{m i}(t) \frac{\partial \boldsymbol{v}_{m}(t)}{\partial x_{i}} \cdot \boldsymbol{v}_{m}^{\prime}(t) d x \\
& -v \int_{\Omega} \nabla \boldsymbol{v}_{m}(t): \nabla \boldsymbol{v}_{m}^{\prime}(t) \boldsymbol{d} \boldsymbol{x}+\int_{\Omega} \boldsymbol{f}(t) \cdot \boldsymbol{v}_{m}^{\prime}(t) \boldsymbol{d} \boldsymbol{x} \\
\leq & \sum_{i, j=1}^{n}\left\|v_{m i}(t)\right\|_{L^{4}(\Omega)}\left\|\frac{\partial v_{m j}(t)}{\partial x_{i}}\right\|_{L^{2}(\Omega)}\left\|v_{m j}^{\prime}(t)\right\|_{L^{4}(\Omega)} \\
& +v\left\|\nabla \boldsymbol{v}_{m}(t)\right\|_{L^{2}(\Omega)}\left\|\nabla \boldsymbol{v}_{m}^{\prime}(t)\right\|_{L^{2}(\Omega)}+\|\boldsymbol{f}(t)\|_{L^{2}(\Omega)}\left\|\boldsymbol{v}_{m}^{\prime}(t)\right\|_{L^{2}(\Omega)} \\
\leq & C_{3}\left(\left\|\boldsymbol{v}_{m}(t)\right\|_{\boldsymbol{V}^{1}(\Omega)}^{2}+\|\boldsymbol{f}(t)\|_{L^{2}(\Omega)}\right)\left\|\boldsymbol{v}_{m}^{\prime}(t)\right\|_{\boldsymbol{V}^{1}(\Omega)^{\prime}}
\end{aligned}
$$

whence

$$
\left\|\boldsymbol{v}_{m}^{\prime}(t)\right\|_{V^{1}(\Omega)} \leq C_{3}\left(\left\|\boldsymbol{v}_{m}(t)\right\|_{V^{1}(\Omega)}^{2}+\|f(t)\|_{L^{2}(\Omega)}\right), \quad t \in(0, T) .
$$

With the help of inequality (16), we get

$$
\left\|\boldsymbol{v}_{m}^{\prime}(t)\right\|_{V^{1}(\Omega)} \leq C_{3}\left(C_{1}\left\|u_{0}\right\|_{V^{2}(\Omega)}^{2}+\int_{0}^{T}\|f(\tau)\|_{L^{2}(\Omega)}^{2} d \tau\right) \exp \left(C_{2} t\right)+C_{3}\|f(t)\|_{L^{2}(\Omega)},
$$

for all $t \in(0, T)$. Therefore, we have

$$
\begin{aligned}
\left\|\boldsymbol{v}_{m}^{\prime}\right\|_{C\left([0, T] ; V^{1}(\Omega)\right)} & =\max _{t \in[0, T]}\left\|\boldsymbol{v}_{m}^{\prime}(t)\right\|_{V^{1}(\Omega)} \\
& \leq C_{3}\left(C_{1}\left\|\boldsymbol{u}_{0}\right\|_{V^{2}(\Omega)}^{2}+\int_{0}^{T}\|f(\tau)\|_{L^{2}(\Omega)}^{2} d \tau\right) \exp \left(C_{2} T\right)+C_{3} \max _{t \in[0, T]}\|f(t)\|_{L^{2}(\Omega)} .
\end{aligned}
$$

We now multiply the $j$ th equation of (12) by $-\lambda_{j} g_{m j}(t)$ and sum with respect to $j$ from 1 to $m$. Taking into account equality (11), we get 


$$
\begin{aligned}
\int_{\Omega} \boldsymbol{v}_{m}^{\prime}(t) \cdot \mathbb{P} \Delta \boldsymbol{v}_{m}(t) d x+\sum_{i=1}^{n} \int_{\Omega} v_{m i}(t) \frac{\partial v_{m}(t)}{\partial x_{i}} \cdot \mathbb{P} \Delta \boldsymbol{v}_{m}(t) d x-v \int_{\Omega} \Delta \boldsymbol{v}_{m}(t) \cdot \mathbb{P} \Delta \boldsymbol{v}_{m}(t) d x \\
\quad-\alpha^{2} \int_{\Omega} \Delta \boldsymbol{v}_{m}^{\prime}(t) \cdot \mathbb{P} \Delta \boldsymbol{v}_{m}(t) d x=\int_{\Omega} f(t) \cdot \mathbb{P} \Delta \boldsymbol{v}_{m}(t) d x, \quad t \in(0, T),
\end{aligned}
$$

which leads to

$$
\begin{aligned}
\int_{\Omega} \boldsymbol{v}_{m}^{\prime}(t) \cdot \mathbb{P} \Delta \boldsymbol{v}_{m}(t) d x+\sum_{i=1}^{n} \int_{\Omega} v_{m i}(t) \frac{\partial \boldsymbol{v}_{m}(t)}{\partial x_{i}} \cdot \mathbb{P} \Delta \boldsymbol{v}_{m}(t) d x-v \int_{\Omega}\left|\mathbb{P} \Delta \boldsymbol{v}_{m}(t)\right|^{2} d x \\
\quad-\alpha^{2} \int_{\Omega} \mathbb{P} \Delta \boldsymbol{v}_{m}^{\prime}(t) \cdot \mathbb{P} \Delta \boldsymbol{v}_{m}(t) d x=\int_{\Omega} f(t) \cdot \mathbb{P} \Delta \boldsymbol{v}_{m}(t) d x, \quad t \in(0, T) .
\end{aligned}
$$

From this equality, with the help of Hölder's and Young's inequalities, we derive

$$
\begin{aligned}
& v\left\|\mathbb{P} \Delta \boldsymbol{v}_{m}(t)\right\|_{L^{2}(\Omega)}^{2}+\frac{\alpha^{2}}{2} \frac{d}{d t}\left\|\mathbb{P} \Delta \boldsymbol{v}_{m}(t)\right\|_{L^{2}(\Omega)}^{2} \\
& =\int_{\Omega} \boldsymbol{v}_{m}^{\prime}(t) \cdot \mathbb{P} \Delta \boldsymbol{v}_{m}(t) d x+\sum_{i=1}^{n} \int_{\Omega} v_{m i}(t) \frac{\partial \boldsymbol{v}_{m}(t)}{\partial x_{i}} \cdot \mathbb{P} \Delta \boldsymbol{v}_{m}(t) d x-\int_{\Omega} f(t) \cdot \mathbb{P} \Delta \boldsymbol{v}_{m}(t) d x \\
& \leq\left(\left\|\boldsymbol{v}_{m}^{\prime}(t)\right\|_{L^{2}(\Omega)}+\sum_{i=1}^{n}\left\|v_{m i}(t)\right\|_{L^{4}(\Omega)}\left\|\frac{\partial v_{m}(t)}{\partial x_{i}}\right\|_{L^{4}(\Omega)}+\|f(t)\|_{L^{2}(\Omega)}\right)\left\|\mathbb{P} \Delta \boldsymbol{v}_{m}(t)\right\|_{L^{2}(\Omega)} \\
& \leq \frac{1}{2 v}\left(\left\|\boldsymbol{v}_{m}^{\prime}(t)\right\|_{L^{2}(\Omega)}+\sum_{i=1}^{n}\left\|v_{m i}(t)\right\|_{L^{4}(\Omega)}\left\|\frac{\partial v_{m}(t)}{\partial x_{i}}\right\|_{L^{4}(\Omega)}+\|f(t)\|_{L^{2}(\Omega)}\right)^{2} \\
& \quad+\frac{v}{2}\left\|\mathbb{P} \Delta v_{m}(t)\right\|_{L^{2}(\Omega)^{\prime}}^{2} \quad t \in(0, T) .
\end{aligned}
$$

Therefore, the following inequality holds

$$
\begin{aligned}
& v\left\|\mathbb{P} \Delta \boldsymbol{v}_{m}(t)\right\|_{L^{2}(\Omega)}^{2}+\alpha^{2} \frac{d}{d t}\left\|\mathbb{P} \Delta \boldsymbol{v}_{m}(t)\right\|_{L^{2}(\Omega)}^{2} \\
& \quad \leq \frac{1}{v}\left(\left\|\boldsymbol{v}_{m}^{\prime}(t)\right\|_{L^{2}(\Omega)}+\sum_{i=1}^{n}\left\|v_{m i}(t)\right\|_{L^{4}(\Omega)}\left\|\frac{\partial v_{m}(t)}{\partial x_{i}}\right\|_{L^{4}(\Omega)}+\|f(t)\|_{L^{2}(\Omega)}\right)^{2}, \quad t \in(0, T),
\end{aligned}
$$

and, using estimates (17) and (18), we deduce that

$$
v\left\|\boldsymbol{v}_{m}(\tau)\right\|_{V^{2}(\Omega)}^{2}+\alpha^{2} \frac{d}{d \tau}\left\|\boldsymbol{v}_{m}(\tau)\right\|_{V^{2}(\Omega)}^{2} \leq C_{4}+C_{5}\left\|\boldsymbol{v}_{m}(\tau)\right\|_{V^{2}(\Omega)^{\prime}}^{2} \quad \tau \in(0, T) .
$$

Integrating both sides of this differential inequality with respect to $\tau$ from 0 to $t$, we deduce

$$
\begin{aligned}
v \int_{0}^{t}\left\|\boldsymbol{v}_{m}(\tau)\right\|_{V^{2}(\Omega)}^{2} d \tau+\alpha^{2}\left\|\boldsymbol{v}_{m}(t)\right\|_{V^{2}(\Omega)}^{2} & \leq \alpha^{2}\left\|\boldsymbol{v}_{m}(0)\right\|_{V^{2}(\Omega)}^{2}+C_{4} t+C_{5} \int_{0}^{t}\left\|\boldsymbol{v}_{m}(\tau)\right\|_{V^{2}(\Omega)}^{2} d \tau \\
& \leq \alpha^{2}\left\|\boldsymbol{u}_{0}\right\|_{V^{2}(\Omega)}^{2}+C_{4} T+C_{5} \int_{0}^{t}\left\|\boldsymbol{v}_{m}(\tau)\right\|_{V^{2}(\Omega)}^{2} d \tau .
\end{aligned}
$$

It follows easily that

$$
\left\|\boldsymbol{v}_{m}(t)\right\|_{V^{2}(\Omega)}^{2} \leq\left\|\boldsymbol{u}_{0}\right\|_{V^{2}(\Omega)}^{2}+C_{4} \alpha^{-2} T+C_{5} \alpha^{-2} \int_{0}^{t}\left\|\boldsymbol{v}_{m}(\tau)\right\|_{V^{2}(\Omega)}^{2} d \tau, \quad t \in(0, T) .
$$


Applying Grönwall's inequality, we obtain

$$
\left\|\boldsymbol{v}_{m}(t)\right\|_{V^{2}(\Omega)}^{2} \leq\left(\left\|\boldsymbol{u}_{0}\right\|_{V^{2}(\Omega)}^{2}+C_{4} \alpha^{-2} T\right) \exp \left(C_{5} \alpha^{-2} t\right), \quad t \in(0, T) .
$$

This implies that

$$
\left\|\boldsymbol{v}_{m}\right\|_{\boldsymbol{C}\left([0, T] ; V^{2}(\Omega)\right)}=\max _{t \in[0, T]}\left\|\boldsymbol{v}_{m}(t)\right\|_{V^{2}(\Omega)} \leq\left\{\left(\left\|\boldsymbol{u}_{0}\right\|_{V^{2}(\Omega)}^{2}+C_{4} \alpha^{-2} T\right) \exp \left(C_{5} \alpha^{-2} T\right)\right\}^{1 / 2} .
$$

Finally, we multiply the $j$ th equation of (12) by $-\lambda_{j} g_{m j}^{\prime}(t)$ and sum with respect to $j$ from 1 to $m$. Bearing in mind equality (11), we obtain

$$
\begin{aligned}
\int_{\Omega} v_{m}^{\prime}(t) \cdot \mathbb{P} \Delta \boldsymbol{v}_{m}^{\prime}(t) d x+\sum_{i=1}^{n} \int_{\Omega} v_{m i}(t) \frac{\partial v_{m}(t)}{\partial x_{i}} \cdot \mathbb{P} \Delta v_{m}^{\prime}(t) d x-v \int_{\Omega} \Delta v_{m}(t) \cdot \mathbb{P} \Delta v_{m}^{\prime}(t) d x \\
-\alpha^{2} \int_{\Omega} \Delta v_{m}^{\prime}(t) \cdot \mathbb{P} \Delta v_{m}^{\prime}(t) d x=\int_{\Omega} f(t) \cdot \mathbb{P} \Delta v_{m}^{\prime}(t) d x, \quad t \in(0, T) .
\end{aligned}
$$

Using Hölder's inequality, from the last equality one can derive

$$
\begin{aligned}
\alpha^{2}\left\|\mathbb{P} \Delta \boldsymbol{v}_{m}^{\prime}(t)\right\|_{L^{2}(\Omega)}^{2}= & \int_{\Omega} \boldsymbol{v}_{m}^{\prime}(t) \cdot \mathbb{P} \Delta \boldsymbol{v}_{m}^{\prime}(t) d x+\sum_{i=1}^{n} \int_{\Omega} v_{m i}(t) \frac{\partial v_{m}(t)}{\partial x_{i}} \cdot \mathbb{P} \Delta \boldsymbol{v}_{m}^{\prime}(t) d x \\
& -v \int_{\Omega} \mathbb{P} \Delta \boldsymbol{v}_{m}(t) \cdot \mathbb{P} \Delta \boldsymbol{v}_{m}^{\prime}(t) d x-\int_{\Omega} f(t) \cdot \mathbb{P} \Delta \boldsymbol{v}_{m}^{\prime}(t) d x \\
\leq & \left(\left\|\boldsymbol{v}_{m}^{\prime}(t)\right\|_{L^{2}(\Omega)}+\sum_{i=1}^{n}\left\|v_{m i}(t)\right\|_{L^{4}(\Omega)}\left\|\frac{\partial v_{m}(t)}{\partial x_{i}}\right\|_{L^{4}(\Omega)}\right. \\
& \left.+v\left\|\mathbb{P} \Delta \boldsymbol{v}_{m}(t)\right\|_{L^{2}(\Omega)}+\|f(t)\|_{L^{2}(\Omega)}\right)\left\|\mathbb{P} \Delta \boldsymbol{v}_{m}^{\prime}(t)\right\|_{L^{2}(\Omega)} \\
\leq & \left(\left\|\boldsymbol{v}_{m}^{\prime}(t)\right\|_{L^{2}(\Omega)}+C_{6}\left\|\boldsymbol{v}_{m}(t)\right\|_{V^{1}(\Omega)}\left\|\boldsymbol{v}_{m}(t)\right\|_{V^{2}(\Omega)}\right. \\
& \left.+v\left\|\boldsymbol{v}_{m}(t)\right\|_{V^{2}(\Omega)}+\|f(t)\|_{L^{2}(\Omega)}\right)\left\|\mathbb{P} \Delta \boldsymbol{v}_{m}^{\prime}(t)\right\|_{L^{2}(\Omega)^{\prime}} \quad t \in(0, T) .
\end{aligned}
$$

Clearly, this yields the estimate

$$
\begin{aligned}
\left\|\mathbb{P} \Delta \boldsymbol{v}_{m}^{\prime}(t)\right\|_{L^{2}(\Omega)} \leq & \alpha^{-2}\left(\left\|\boldsymbol{v}_{m}^{\prime}(t)\right\|_{L^{2}(\Omega)}+C_{6}\left\|\boldsymbol{v}_{m}(t)\right\|_{V^{1}(\Omega)}\left\|\boldsymbol{v}_{m}(t)\right\|_{V^{2}(\Omega)}\right. \\
& \left.+v\left\|\boldsymbol{v}_{m}(t)\right\|_{V^{2}(\Omega)}+\|f(t)\|_{L^{2}(\Omega)}\right), \quad t \in(0, T) .
\end{aligned}
$$

Taking into account (17)-(19), from the last inequality, we easily obtain that

$$
\left\|\boldsymbol{v}_{m}^{\prime}(t)\right\|_{V^{2}(\Omega)}=\left\|\mathbb{P} \Delta \boldsymbol{v}_{m}^{\prime}(t)\right\|_{L^{2}(\Omega)} \leq C_{7}, \quad t \in(0, T),
$$

and, hence,

$$
\left\|\boldsymbol{v}_{m}^{\prime}\right\|_{C\left([0, T] ; V^{2}(\Omega)\right)}=\max _{t \in[0, T]}\left\|\boldsymbol{v}_{m}^{\prime}(t)\right\|_{V^{2}(\Omega)} \leq C_{7}
$$

From estimates (19) and (20) and Lemma 2, it follows that there exist a subsequence $\left\{m_{k}\right\}_{k=1}^{\infty}$ and a function $\boldsymbol{u}$ such that $\boldsymbol{v}_{m_{k}}$ converges strongly to $\boldsymbol{u}$ in the space $\boldsymbol{C}\left([0, T] ; \boldsymbol{V}^{1}(\Omega)\right)$ as $k \rightarrow \infty$. Without loss of generality, we can assume that 


$$
\begin{aligned}
& \boldsymbol{v}_{m} \rightarrow \boldsymbol{u} \text { strongly in } \boldsymbol{C}\left([0, T] ; \boldsymbol{V}^{1}(\Omega)\right) \text { as } m \rightarrow \infty, \\
& \boldsymbol{v}_{m} \rightarrow \boldsymbol{u} \text { weakly in } \boldsymbol{L}^{2}\left(0, T ; \boldsymbol{V}^{2}(\Omega)\right) \text { as } m \rightarrow \infty .
\end{aligned}
$$

Moreover, we have

$$
\boldsymbol{v}_{m}(0) \rightarrow \boldsymbol{u}_{0} \text { strongly in } \boldsymbol{V}^{2}(\Omega) \text { as } m \rightarrow \infty .
$$

On the other hand, from (21) it follows that

$$
\boldsymbol{v}_{m}(0) \rightarrow \boldsymbol{u}(0) \text { strongly in } \boldsymbol{V}^{1}(\Omega) \text { as } m \rightarrow \infty \text {. }
$$

Comparing the convergence results (23) and (24), we obtain

$$
\boldsymbol{u}(0)=\boldsymbol{u}_{0}
$$

Integrating the $j$ th equation of (12) from 0 to $s$, we obtain

$$
\begin{array}{r}
\int_{\Omega} \boldsymbol{v}_{m}(s) \cdot \boldsymbol{w}_{j} d x+\sum_{i=1}^{n} \int_{0}^{s} \int_{\Omega} v_{m i}(t) \frac{\partial \boldsymbol{v}_{m}(t)}{\partial x_{i}} \cdot \boldsymbol{w}_{j} d x d t-v \int_{0}^{s} \int_{\Omega} \Delta \boldsymbol{v}_{m}(t) \cdot \boldsymbol{w}_{j} d x d t-\alpha^{2} \int_{\Omega} \Delta \boldsymbol{v}_{m}(s) \cdot \boldsymbol{w}_{j} d x \\
=\int_{\Omega} \boldsymbol{v}_{m}(0) \cdot \boldsymbol{w}_{j} d x-\alpha^{2} \int_{\Omega} \Delta \boldsymbol{v}_{m}(0) \cdot \boldsymbol{w}_{j} d x+\int_{0} \int_{\Omega} f(t) \cdot \boldsymbol{w}_{j} d x d t, \quad j \in\{1,2, \ldots\}, s \in[0, T] .
\end{array}
$$

Integrating by parts the third and fourth terms on the left-hand side of this equality, we arrive at

$$
\begin{aligned}
& \int_{\Omega} \boldsymbol{v}_{m}(s) \cdot \boldsymbol{w}_{j} \boldsymbol{d} \boldsymbol{x}+\sum_{i=1}^{n} \int_{0}^{s} \int_{\Omega} v_{m i}(t) \frac{\partial \boldsymbol{v}_{m}(t)}{\partial x_{i}} \cdot \boldsymbol{w}_{j} d x d t+v \int_{0}^{s} \int_{\Omega} \nabla \boldsymbol{v}_{m}(t): \nabla \boldsymbol{w}_{j} d x d t \\
& \quad+\alpha^{2} \int_{\Omega} \nabla \boldsymbol{v}_{m}(s): \nabla \boldsymbol{w}_{j} d x=\int_{\Omega} \boldsymbol{v}_{m}(0) \cdot \boldsymbol{w}_{j} d x-\alpha^{2} \int_{\Omega} \Delta \boldsymbol{v}_{m}(0) \cdot \boldsymbol{w}_{j} d x+\int_{0}^{s} \int_{\Omega} f(t) \cdot \boldsymbol{w}_{j} d x d t
\end{aligned}
$$

Using the convergence results (21)-(23), we can pass to the limit $m \rightarrow \infty$ in the last equality and obtain

$$
\begin{aligned}
\int_{\Omega} \boldsymbol{u}(s) \cdot \boldsymbol{w}_{j} d x & +\sum_{i=1}^{n} \int_{0}^{s} \int_{\Omega} u_{i}(t) \frac{\partial \boldsymbol{u}(t)}{\partial x_{i}} \cdot \boldsymbol{w}_{j} d x d t+v \int_{0} \int_{\Omega} \nabla \boldsymbol{u}(t): \nabla \boldsymbol{w}_{j} d x d t+\alpha^{2} \int_{\Omega} \nabla \boldsymbol{u}(s): \nabla \boldsymbol{w}_{j} d \boldsymbol{x} \\
& =\int_{\Omega} \boldsymbol{u}_{0} \cdot \boldsymbol{w}_{j} d x-\alpha^{2} \int_{\Omega} \Delta \boldsymbol{u}_{0} \cdot \boldsymbol{w}_{j} d x+\int_{0} \int_{\Omega} f(t) \cdot \boldsymbol{w}_{j} d x d t, \quad j \in\{1,2, \ldots\}, s \in[0, T] .
\end{aligned}
$$

Applying integration by parts again, we get

$$
\begin{array}{r}
\int_{\Omega} u(s) \cdot w_{j} d x+\sum_{i=1}^{n} \int_{0}^{s} \int_{\Omega} u_{i}(t) \frac{\partial u(t)}{\partial x_{i}} \cdot w_{j} d x d t-v \int_{0} \int_{\Omega} \Delta u(t) \cdot w_{j} d x d t-\alpha^{2} \int_{\Omega} \Delta u(s) \cdot w_{j} d x \\
=\int_{\Omega} u_{0} \cdot w_{j} d x-\alpha^{2} \int_{\Omega} \Delta u_{0} \cdot w_{j} d x+\int_{0} \int_{\Omega} f(t) \cdot w_{j} d x d t, \quad j \in\{1,2, \ldots\}, s \in[0, T] .
\end{array}
$$


Because $\left\{\boldsymbol{w}_{j}\right\}_{j=1}^{\infty}$ is a basis of $\boldsymbol{V}^{0}(\Omega)$, equality (26) remains valid if we replace $\boldsymbol{w}_{j}$ with an arbitrary vector function $w$ from the space $V^{0}(\Omega)$, that is

$$
\begin{aligned}
\int_{\Omega} \boldsymbol{u}(s) \cdot \boldsymbol{w} d x & +\sum_{i=1}^{n} \int_{0}^{s} \int_{\Omega} u_{i}(t) \frac{\partial \boldsymbol{u}(t)}{\partial x_{i}} \cdot \boldsymbol{w} d x d t-v \int_{0}^{s} \int_{\Omega} \Delta \boldsymbol{u}(t) \cdot \boldsymbol{w} d x d t \\
& -\alpha^{2} \int_{\Omega} \Delta \boldsymbol{u}(s) \cdot \boldsymbol{w} d x=\int_{\Omega} \boldsymbol{u}_{0} \cdot \boldsymbol{w} d x-\alpha^{2} \int_{\Omega} \Delta \boldsymbol{u}_{0} \cdot \boldsymbol{w} d x+\int_{0}^{s} \int_{\Omega} f(t) \cdot \boldsymbol{w} d x d t, \quad s \in[0, T] .
\end{aligned}
$$

From the last equality it follows that

$$
\boldsymbol{u}(s)+\sum_{i=1}^{n} \mathbb{P} \int_{0}^{s} u_{i}(t) \frac{\partial \boldsymbol{u}(t)}{\partial x_{i}} d t-v \mathbb{P} \int_{0}^{s} \Delta \boldsymbol{u}(t) d t-\alpha^{2} \mathbb{P} \Delta \boldsymbol{u}(s)=\boldsymbol{u}_{0}-\alpha^{2} \mathbb{P} \Delta \boldsymbol{u}_{0}+\mathbb{P} \int_{0}^{s} \boldsymbol{f}(t) d t
$$

Using the Stokes operator $\mathbb{A}$, we can rewrite this equality as follows

$$
\left(\mathbb{I}+\alpha^{2} \mathbb{A}\right) \boldsymbol{u}(s)=-\sum_{i=1}^{n} \mathbb{P} \int_{0}^{s} u_{i}(t) \frac{\partial \boldsymbol{u}(t)}{\partial x_{i}} d t+v \mathbb{P} \int_{0}^{s} \Delta \boldsymbol{u}(t) d t+\left(\mathbb{I}+\alpha^{2} \mathbb{A}\right) \boldsymbol{u}_{0}+\mathbb{P} \int_{0}^{s} \boldsymbol{f}(t) d t, \quad s \in[0, T],
$$

where $\mathbb{I}: V^{2}(\Omega) \rightarrow V^{0}(\Omega)$ is the embedding operator.

Applying the operator $\left(\mathbb{I}+\alpha^{2} \mathbb{A}\right)^{-1}: V^{0}(\Omega) \rightarrow V^{2}(\Omega)$ to both sides of equality (28), we get

$$
\begin{aligned}
\boldsymbol{u}(s)= & -\sum_{i=1}^{n}\left(\mathbb{I}+\alpha^{2} \mathbb{A}\right)^{-1} \mathbb{P} \int_{0}^{s} u_{i}(t) \frac{\partial \boldsymbol{u}(t)}{\partial x_{i}} d t+v\left(\mathbb{I}+\alpha^{2} \mathbb{A}\right)^{-1} \mathbb{P} \int_{0}^{s} \Delta \boldsymbol{u}(t) d t \\
& +\boldsymbol{u}_{0}+\left(\mathbb{I}+\alpha^{2} \mathbb{A}\right)^{-1} \mathbb{P} \int_{0}^{s} f(t) d t, \quad s \in[0, T] .
\end{aligned}
$$

Since

$$
\boldsymbol{u} \in \boldsymbol{C}\left([0, T] ; \boldsymbol{V}^{1}(\Omega)\right) \cap \boldsymbol{L}^{2}\left(0, T ; \boldsymbol{V}^{2}(\Omega)\right),
$$

we conclude from (29) that

$$
\boldsymbol{u} \in \boldsymbol{C}\left([0, T] ; \boldsymbol{V}^{2}(\Omega)\right) .
$$

Next, differentiating both sides of (29) with respect to $s$, we get

$$
\boldsymbol{u}^{\prime}(s)=-\sum_{i=1}^{n}\left(\mathbb{I}+\alpha^{2} \mathbb{A}\right)^{-1} \mathbb{P}\left[u_{i}(s) \frac{\partial \boldsymbol{u}(s)}{\partial x_{i}}\right]+v\left(\mathbb{I}+\alpha^{2} \mathbb{A}\right)^{-1} \mathbb{P} \Delta \boldsymbol{u}(s)+\left(\mathbb{I}+\alpha^{2} \mathbb{A}\right)^{-1} \mathbb{P} \boldsymbol{f}(s), \quad s \in[0, T]
$$

Taking into account (30), from the last equality we deduce that $\boldsymbol{u}^{\prime} \in \mathcal{C}\left([0, T] ; \boldsymbol{V}^{2}(\Omega)\right)$. Hence,

$$
u \in C^{1}\left([0, T] ; V^{2}(\Omega)\right) .
$$

Next, from equality (27) it follows that there exists an element $\bar{\pi}(t) \in H^{1}(\Omega) / \mathbb{R}$ such that

$$
\boldsymbol{u}(t)+\sum_{i=1}^{n} \int_{0}^{t} u_{i}(\tau) \frac{\partial \boldsymbol{u}(\tau)}{\partial x_{i}} d \tau-v \int_{0}^{t} \Delta \boldsymbol{u}(\tau) d \tau-\alpha^{2} \Delta \boldsymbol{u}(t)-\boldsymbol{u}_{0}-\alpha^{2} \Delta \boldsymbol{u}_{0}-\int_{0}^{t} f(\tau) d \tau=\nabla \bar{\pi}(t)
$$

It is readily seen that $\nabla \bar{\pi} \in C^{1}([0, T] ; G(\Omega))$ and, consequently, we have

$$
\bar{\pi} \in C^{1}\left([0, T] ; H^{1}(\Omega) / \mathbb{R}\right) .
$$


Letting $\bar{p}(t) \stackrel{\text { def }}{=}-\bar{\pi}^{\prime}(t)$, from (33) we get

$$
\bar{p} \in C\left([0, T] ; H^{1}(\Omega) / \mathbb{R}\right) .
$$

Finally, differentiating both sides of (32) with respect to $t$, we arrive at

$$
\boldsymbol{u}^{\prime}(t)+\sum_{i=1}^{n} u_{i}(t) \frac{\partial \boldsymbol{u}(t)}{\partial x_{i}}-v \Delta \boldsymbol{u}(t)-\alpha^{2} \Delta \boldsymbol{u}^{\prime}(t)+\nabla \bar{p}(t)=\boldsymbol{f}(t), \quad t \in(0, T) .
$$

Bearing in mind (25), (31), (34), and (35), we conclude that the pair $(\boldsymbol{u}, \bar{p})$ is a strong solution to problem (1) on the interval $[0, T]$. The uniqueness of a strong solution can be proved by using arguments similar to those that are presented in [9], thus we choose to omit the details of the corresponding proof. Since $T$ is arbitrary, we see that $(u, \bar{p})$ is a solution of (1) in the sense of Definition 1.

Next, we take the $L^{2}$-scalar product of (8) with the vector function $\boldsymbol{u}$. Using integration by parts, one can easily arrive at the energy equality (9).

The rest of the proof consists in proving inequality (10). If there exists a function $q$ from the space $C\left([0, T] ; H^{1}(\Omega)\right)$ such that $\nabla q=f$, then we have

$$
\int_{\Omega} f(\tau) \cdot \boldsymbol{u}(\tau) d x=\int_{\Omega} \nabla q(\tau) \cdot \boldsymbol{u}(\tau) d x=-\int_{\Omega} q(\tau) \underbrace{\nabla \cdot \boldsymbol{u}(\tau)}_{=0} d x=0, \quad \tau \geq 0,
$$

i. e., the total work done by external forces $f$ is zero.

In view of (36), the energy equality (9) reduces to

$$
\|\boldsymbol{u}(t)\|_{\mathbf{L}^{2}(\Omega)}^{2}+2 v \int_{0}^{t}\|\nabla \boldsymbol{u}(\tau)\|_{\boldsymbol{L}^{2}(\Omega)}^{2} d \tau+\alpha^{2}\|\nabla \boldsymbol{u}(t)\|_{\mathbf{L}^{2}(\Omega)}^{2}=\left\|\boldsymbol{u}_{0}\right\|_{\boldsymbol{L}^{2}(\Omega)}^{2}+\alpha^{2}\left\|\nabla \boldsymbol{u}_{0}\right\|_{\boldsymbol{L}^{2}(\Omega)^{\prime}}^{2} \quad t \geq 0 .
$$

Differentiating the last equality with respect to $t$, we get

$$
\frac{d}{d t}\left[\|\boldsymbol{u}(t)\|_{L^{2}(\Omega)}^{2}+\alpha^{2}\|\nabla \boldsymbol{u}(t)\|_{L^{2}(\Omega)}^{2}\right]+2 v\|\nabla \boldsymbol{u}(t)\|_{L^{2}(\Omega)}^{2}=0, \quad t \geq 0 .
$$

Using inequality (6), we obtain

$$
\frac{d}{d t}\left[\|\boldsymbol{u}(t)\|_{L^{2}(\Omega)}^{2}+\alpha^{2}\|\nabla \boldsymbol{u}(t)\|_{L^{2}(\Omega)}^{2}\right]+\frac{8 v}{d_{\Omega}^{2}+4 \alpha^{2}}\left[\|\boldsymbol{u}(t)\|_{L^{2}(\Omega)}^{2}+\alpha^{2}\|\nabla \boldsymbol{u}(t)\|_{L^{2}(\Omega)}^{2}\right] \leq 0, \quad t \geq 0
$$

and, hence,

$$
\frac{d}{d t}\left[\exp \left(\frac{8 v t}{d_{\Omega}^{2}+4 \alpha^{2}}\right)\left\{\|\boldsymbol{u}(t)\|_{L^{2}(\Omega)}^{2}+\alpha^{2}\|\nabla \boldsymbol{u}(t)\|_{L^{2}(\Omega)}^{2}\right\}\right] \leq 0, \quad t \geq 0 .
$$

Then, by integrating (37) with respect to $t$, we derive inequality (10). Thus, the proof of Theorem 1 is complete.

\section{Concluding Remarks}

In this paper, we prove the existence and uniqueness of a strong solution to the incompressible Navier-Stokes-Voigt model. The construction of a strong solution proceeds via the Faedo-Galerkin procedure with a special basis of eigenfunctions of the Stokes operator. Note that this approach allows easily obtaining approximations of strong solutions, which frequently reduce to approximate analytic or semi-analytic solutions when the flow domain has a simple symmetric shape. Such solutions favor a better understanding of the qualitative features of unsteady flows of viscoelastic fluids and can be used to test the relevant numerical, asymptotic, and approximate methods. 
Funding: This research received no external funding.

Conflicts of Interest: The author declares no conflict of interest.

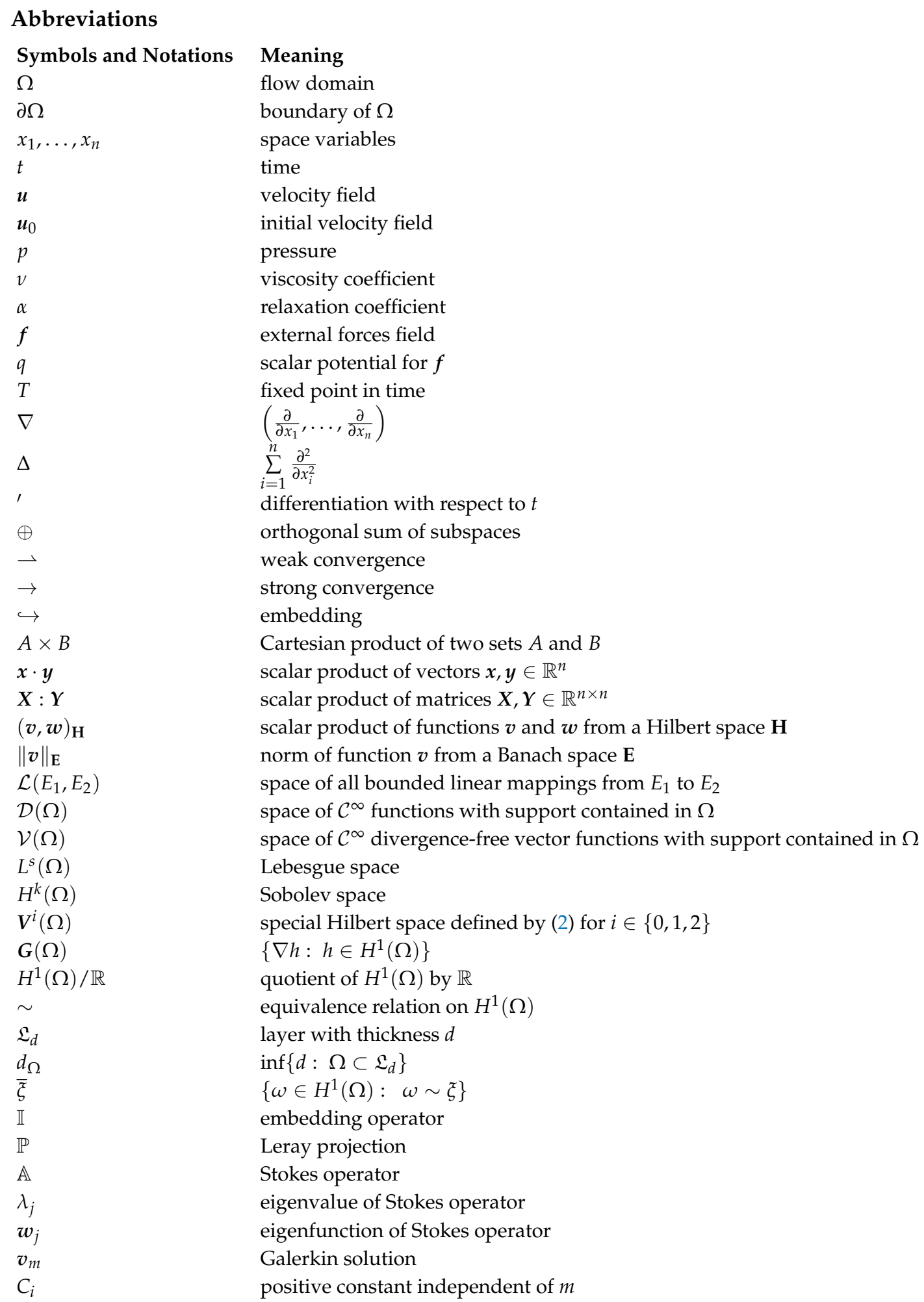




\section{References}

1. Oskolkov, A.P. Solvability in the large of the first boundary value problem for a certain quasilinear third order system that is encountered in the study of the motion of a viscous fluid. Zap. Nauchn. Semin. LOMI 1972, 27, 145-160.

2. Oskolkov, A.P. On the uniqueness and solvability in the large of the boundary-value problems for the equations of motion of aqueous solutions of polymers. Zap. Nauchn. Semin. LOMI 1973, 38, 98-136.

3. Oskolkov, A.P. Some quasilinear systems that arise in the study of the motion of viscous fluids. Zap. Nauchn. Semin. LOMI 1975, 52, 128-157

4. Ladyzhenskaya, O.A. On some gaps in two of my papers on the Navier-Stokes equations and the way of closing them. J. Math. Sci. 2003, 115, 2789-2791. [CrossRef]

5. Sviridyuk, G.A. On a model of the dynamics of a weakly compressible viscoelastic fluid. Russian Math. (Iz. VUZ) 1994, 38, 59-68.

6. Sviridyuk, G.A.; Sukacheva T.G. On the solvability of a nonstationary problem describing the dynamics of an incompressible viscoelastic fluid. Math. Notes 1998, 63, 388-395. [CrossRef]

7. Korpusov, M.O.; Sveshnikov, A.G. Blow-up of Oskolkov's system of equations. Sb. Math. 2009, 200, 549-572. [CrossRef]

8. Ladyzhenskaya, O.A. On the global unique solvability of some two-dimensional problems for the water solutions of polymers. J. Math. Sci. 2000, 99, 888-897. [CrossRef]

9. Baranovskii, E.S. Mixed initial-boundary value problem for equations of motion of Kelvin-Voigt fluids. Comput. Math. Math. Phys. 2016, 56, 1363-1371. [CrossRef]

10. Baranovskii, E.S. Global solutions for a model of polymeric flows with wall slip. Math. Meth. Appl. Sci. 2017, 40, 5035-5043. [CrossRef]

11. Kaya, M.; Celebi, A.O. Existence of weak solutions of the g-Kelvin-Voight equation. Math. Comput. Model. 2009, 49, 497-504. [CrossRef]

12. Baranovskii, E.S. Flows of a polymer fluid in domain with impermeable boundaries. Comput. Math. Math. Phys. 2014, 54, 1589-1596. [CrossRef]

13. Berselli, L.C.; Spirito, S. Suitable weak solutions to the 3D Navier-Stokes equations are constructed with the Voigt approximation. J. Differ. Equ. 2017, 262, 3285-3316. [CrossRef]

14. Caffarelli, L.; Kohn, R.; Nirenberg, L. Partial regularity of suitable weak solutions of the Navier-Stokes equations. Comm. Pure Appl. Math. 1982, 35, 771-831. [CrossRef]

15. Fedorov, V.E.; Ivanova, N.D. Inverse problem for Oskolkov's system of equations. Math. Meth. Appl. Sci. 2017, 40, 6123-6126. [CrossRef]

16. Plekhanova, M.V.; Baybulatova, G.D.; Davydov, P.N. Numerical solution of an optimal control problem for Oskolkov's system. Math. Meth. Appl. Sci. 2018, 41, 9071-9080. [CrossRef]

17. Antontsev, S.N.; Khompysh, K. Kelvin-Voight equation with p-Laplacian and damping term: Existence, uniqueness and blow-up. J. Math. Anal. Appl. 2017, 446, 1255-1273. [CrossRef]

18. Artemov, M.A.; Baranovskii, E.S. Solvability of the Boussinesq approximation for water polymer solutions. Mathematics 2019, 7, 611. [CrossRef]

19. Mohan, M.T. On the three dimensional Kelvin-Voigt fluids: Global solvability, exponential stability and exact controllability of Galerkin approximations. Evol. Equ. Control Theory 2019. [CrossRef]

20. Boyer, F.; Fabrie, P. Mathematical Tools for the Study of the Incompressible Navier-Stokes Equations and Related Models; Springer: New York, NY, USA, 2013. [CrossRef]

21. Temam, R. Navier-Stokes Equations-Theory and Numerical Analysis; North-Holland Publishing Co.: Amsterdam, The Netherlands, 1977.

22. Simon, J. Compact sets in the space $L^{p}(0, T ; B)$. Ann. Mat. Pura Appl. 1986, 146, 65-96. [CrossRef]

23. Agranovich, M.S. Sobolev Spaces, Their Generalizations, and Elliptic Problems in Smooth and Lipschitz Domains; Springer: Cham, Switzerland, 2015. [CrossRef] 
24. Galdi, G.P. An Introduction to the Mathematical Theory of the Navier-Stokes Equations-Steady-State Problems; Springer: New York, NY, USA, 2011. [CrossRef]

25. Siddiqi, A.H. Functional Analysis and Applications; Springer: Singapore, 2018. [CrossRef]

(C) 2020 by the author. Licensee MDPI, Basel, Switzerland. This article is an open access article distributed under the terms and conditions of the Creative Commons Attribution (CC BY) license (http://creativecommons.org/licenses/by/4.0/). 